\title{
Thiamine Therapy for Dystonia Type 1
}

\author{
Costantini Antonio ${ }^{1,2^{*}}$, Pala Maria Immacolata ${ }^{3}$, Trevi Erika ${ }^{2}$, Maurini Serena ${ }^{2}$, Vecchietti \\ Stefania $^{2}$, Fancellu Roberto ${ }^{4}$ \\ ${ }^{1}$ Università Cattolica di Roma, Largo Agostino Gemelli, Roma, Italy \\ ${ }^{2}$ Polispecialistic Center "Giovanni Paolo I"- Viterbo, Italy \\ ${ }^{3}$ Department of Neurological Rehabilitation of the Clinic "Villa Immacolata" - Viterbo, Italy \\ ${ }^{4}$ Unit of Neurology, San Martino IRCCS Policlinic Hospital, Genova, Italy
}

*Corresponding Author: Costantini Antonio, Università Cattolica di Roma, Largo Agostino Gemelli, Roma, Italy, Email: carapetata@libero.it

\begin{abstract}
Dystonia type 1 is an early-onset inherited dystonia presents in childhood or adolescence. Dystonia type 1 is characterized by involuntary, repetitive, sustained muscle contractions or postures involving one or more sites of the body in absence of other neurological symptoms. Symptoms develop first in an arm or leg in middle to late childhood and can extend to other body regions. In this report two patients affected by Dystonia 1 began an intramuscular therapy with high-dose thiamine from April 2016. This therapy was effective in improving the motor problems. Our results support the hypothesis that Dystonia 1 might have a mitochondrial etiology.
\end{abstract}

Keywords: Dystonia 1, Dystonia 1 and Thiamine, Dystonia 1 Therapy, High-Dose Thiamine.

\section{INTRODUCTION}

Primary torsion dystonia (PTD) is characterized by sustained muscle contractions, frequently causing repetitive twisting movements or abnormal postures. In PTD, dystonia is the only clinical sign. Primary forms of dystonia are caused by neuronal dysfunction but not neuronal death. The result of the pathogenic changes in primary dystonia is a defect in neural circuits involving the basal ganglia, sensorimotor cortex and cerebellum. Neuroimaging study could not identify structural or functional abnormalities in both focal and generalized PTD. [1]

Currently, effective therapies targeted at the pathogenic mechanism of dystonia are not yet available [1].

PTD include some genetic forms as primary dystonia type 1 (DYT1) which is one of the most common early-onset. The DYT1 is diagnosed by molecular genetic test to TORIA gene deletion which reveals a three-base pair GAG in most affected individuals. DYT1 exhibits autosomal dominant inheritance. This gene encodes a protein named Torsin A, which is presumed to act as a chaperone protein associated with endoplasmic reticulum and the nuclear envelope. Its function remains to be determined $[1,2]$.
Thiamine deficiency (TD) in humans causes beriberi and Wernicke-Korsakoff syndrome [3].

Thiamine plays a fundamental role in energy metabolism and in the maintenance of brain function [3,4]. Recent studies implicate thiamine in oxidative stress, protein processing, peroxisomal function, and gene expression $[4,5]$.

Defects of energetic metabolism have been implicated in a variety of neurodegenerative diseases [6]. Some authors found that high-dose thiamine improves the symptoms of Dystonia 16 and sporadic primary dystonia $[7,8]$.

Movement disorder dystonia has been associated with a dysfunction of carboidrate metabolism of neural cells. In fact, some authors hypothesized the possibility that dystonia might have a mitochondrial etiology [9].

Hypothesizing that a dysfunction of thiaminedependent processes was concerned in the pathogenesis of the symptoms of DYT1, we treated two patients affected by DYT1 (mother and son) with high-dose thiamine to see the effectiveness of this therapy in DYT1.

Written informed consent was taken from the patients. The study did not require approval by Ethic committee as per the local retrospective observations. 


\section{CASE Presentation}

The patients were affected by DYT1. There was not identifiable exogenous causes or other degenerative diseases. The diagnosis of DYT1 was based on clinical history, physical examination, neuroradiological exams and genetic test.

In both the patients, molecular analysis of $D Y T$ 1 gene (performed at Pisa University) showed the presence of deletion of one triplet GAG (c.907_909 of GAG. Ensembl Gene ID ENSG00000136827) in the exon 5 of the DYT 1 gene.

We assessed the patients with dystonia using the Fahn-Marsden Rating Scales (dystonia movement and disability scales). The first patient started the therapy in April 2016 and the second patient in June 2016. The follow-ups have been done after three months of treatment.

Dystonia movement scale (DMS) consists of provoking factors (scored 0-4) and severity factors (scored 0-4). Some are given a weight (W) of $1 / 2$, and others a weight of 1 . All three are multiplied to give the adjusted score; all scores are summed to give an overall score (S) from 0 to 120 [10]

Dystonia disability scale (DDS) is a tool assessing the disability provoked by dystonia in seven items, whose six are scored $0-4$ and the last 0-6 (the gait assessment must consider the possible use of a wheelchair). The total score ranges from 0 to 30 [10].

Pain and fatigue were evaluated using the visual analogical scale (VAS).

Soon after evaluating the patients with the FahnMarsden Rating Scales, the patients began treatment with an intramuscular phial of $100 \mathrm{mg} / \mathrm{ml}$ twice a week.

\section{Patient 1}

Female, 47-year-old, with a weight of $60 \mathrm{Kg}$. The dystonic symptomatology started at the age of 10 with handwriting disorders, which subsequently extended to her lower limbs and to the whole body. The neurological examination was normal except for the dystonic movements. Common biochemical and hematological investigations and neuroradiological exams were normal. Plasmatic thiamine level was 84 $\mathrm{mg} / \mathrm{l}$ (normal values 28-85). She had a generalized dystonia present at rest with speech disorders. She also had eyes dystonia with spasms of eyelid closure, neck dystonia with often obvious mild torticollis and pulling and upper and lower limbs dystonia. This caused handwriting, feeding, dressing and gait disorders. The patient had an appreciable fatigue, a continuous thoracic-scapular pain on the right side of the body. The pain, sometimes, increased so much that she was obliged to rest in bed for a number of days. The severe pain was unresponsive to any kind of treatment.

The score of DMS was 66 and of DDS was 14 (table 1).

The VAS scale before starting the treatment was 50/100 for fatigue and 100/100 for pain.

\section{Patient 2}

Male, 29-year-old, with a weight of $80 \mathrm{Kg}$. The neurological examination was normal except for the dystonic disorder.

Common biochemical and hematological investigations were normal. Plasmatic thiamine level was $75 \mathrm{mg} / \mathrm{l}$ (normal values 28-85). The dystonic symptomatology started at the age of 10 , with slight handwriting and right harm dystonia, with difficulty in the use of the mouse. Later also his right foot presented dystonia with mild supination and dynamic equinus foot deformity during the swing phase of the gait with descent to the ground on the anterior and lateral part of the same foot plant. The gait disturbances got worse after a path of about 100 $\mathrm{m}$ so that we had to ask him to stop.

The score of DMS was 5 and of DDS was 2 (table 1).

After 3 months from the beginning of the therapy, we examined the patients and repeated the same tests.

The patient 1, already few hours after the first thiamine administration, noticed a marked regression of the dystonic symptoms. Afterward, at the end of the treatment fatigue and pain had a complete regression measured with the VAS scale. In particular the patient noticed an appreciable regression of handwriting, feeding, dressing and gait disorders and the patient had an improvement of $56.8 \%$ for the DMS and of $42.8 \%$ for the DDS (Table 1).

The patient 2 started to notice an improvement at the third injection. After 30 days from the beginning of the treatment, he had an appreciable improvement of all the symptoms. He succeeded in walking for $200 \mathrm{~m}$ before stopping (Table 1).

The patient had an improvement of $40.0 \%$ for the DMS and of $50 \%$ for the DDS (Table 1). 
Table1: The Fahn-Marsden dystonia scale scores

\begin{tabular}{|c|c|c|c|c|c|c|c|c|c|c|c|c|c|}
\hline \multirow{2}{*}{\multicolumn{2}{|c|}{ Provoking factors (PF) }} & \multicolumn{3}{|c|}{ Patient 1 before } & \multicolumn{3}{|c|}{ Patient 1 after } & \multicolumn{3}{|c|}{ Patient 2 before } & \multicolumn{3}{|c|}{ Patient 2 after } \\
\hline & & & & \\
\hline \multicolumn{2}{|c|}{ General } & \multicolumn{3}{|l|}{4} & \multicolumn{3}{|l|}{3} & \multicolumn{3}{|l|}{1} & \multicolumn{3}{|l|}{1} \\
\hline \multicolumn{2}{|c|}{ Speech and swallowing (SS) } & \multicolumn{3}{|l|}{2} & \multicolumn{3}{|l|}{1} & \multicolumn{3}{|l|}{0} & \multicolumn{3}{|l|}{0} \\
\hline \multicolumn{14}{|c|}{ Severity factors (SF) } \\
\hline \multicolumn{2}{|l|}{ Eyes } & \multicolumn{3}{|l|}{1} & \multicolumn{3}{|l|}{0} & \multicolumn{3}{|l|}{0} & \multicolumn{3}{|l|}{0} \\
\hline \multicolumn{2}{|l|}{ Mouth } & \multicolumn{3}{|l|}{0} & \multicolumn{3}{|l|}{0} & \multicolumn{3}{|l|}{0} & 0 & & \\
\hline Speech and swallo & ving & 1 & & & 0 & & & 0 & & & 0 & & \\
\hline Neck & & 1 & & & 0 & & & 0 & & & 0 & & \\
\hline Arm & & 3 & & & 2 & & & 2 & & & 1 & & \\
\hline Trunk & & 3 & & & 2 & & & 0 & & & 0 & & \\
\hline Leg & & 3 & & & 3 & & & 3 & & & 2 & & \\
\hline Scoring & $\mathrm{W}$ & PF & SF & Score & $\mathrm{PF}$ & SF & Score & $\mathrm{PF}$ & SF & Score & $\mathrm{PF}$ & $\mathrm{SF}$ & Score \\
\hline Eyes & 0.5 & 4 & 1 & 2 & 3 & 0 & 0 & 1 & 0 & 0 & 1 & 0 & 0 \\
\hline Mouth & 0.5 & 4 & 0 & 0 & 3 & 0 & 0 & 1 & 0 & 0 & 1 & 0 & 0 \\
\hline $\begin{array}{ll}\text { Speech } & \text { and } \\
\text { swallowing }\end{array}$ & 1.0 & 2 & 1 & 2 & 1 & 0 & 0 & 0 & 0 & 0 & 0 & 0 & 0 \\
\hline Neck & 0.5 & 4 & 1 & 2 & 3 & 1 & 1.5 & 1 & 0 & 0 & 1 & 0 & 0 \\
\hline Right Arm & 1.0 & 4 & 3 & 12 & 3 & 3 & 9 & 1 & 2 & 2 & 1 & 1 & 0 \\
\hline Left Arm & 1.0 & 4 & 0 & 0 & 3 & 0 & 0 & 1 & 0 & 0 & 1 & 0 & 0 \\
\hline Trunk & 1.0 & 4 & 3 & 12 & 3 & 3 & 9 & 1 & 0 & 0 & 1 & 0 & 0 \\
\hline Right Leg & 1.0 & 4 & 3 & 12 & 3 & 3 & 9 & 1 & 3 & 3 & 1 & 2 & 2 \\
\hline Left Leg & 1.0 & 4 & 0 & 0 & 3 & 0 & 0 & 1 & 0 & 0 & 1 & 0 & 0 \\
\hline Total Points $(\mathrm{S})$ & & & & 66 & & & 28.5 & & & 5 & & & 2 \\
\hline Dystonia Disability & Scale & & & & & & & & & & & & \\
\hline Speech & & 1 & & & 0 & & & 0 & & & 0 & & \\
\hline Handwriting & & 4 & & & 3 & & & 2 & & & 1 & & \\
\hline Feeding & & 2 & & & 1 & & & 0 & & & 0 & & \\
\hline Eating/Swallowing & & 0 & & & 0 & & & 0 & & & 0 & & \\
\hline Hygiene & & 1 & & & 0 & & & 0 & & & 0 & & \\
\hline Dressing & & 1 & & & 1 & & & 0 & & & 0 & & \\
\hline Walking & & 4 & & & 3 & & & 2 & & & 1 & & \\
\hline Total Points & & 14 & & & 8 & & & 4 & & & 2 & & \\
\hline
\end{tabular}

\section{DISCUSSION}

Injections of high-dose thiamine were effective in improving the motor and non-motor disorders of the patients. This result suggests that the abnormalities in thiamine-dependent processes could be overcome by a diffusion-mediated transport at supra-normal thiamine concentrations. The presence of symptoms due to a thiamine deficiency in patients with normal concentrations of plasmatic thiamine could be explained if referred to a form of thiamine deficiency due to enzymatic abnormalities or to dysfunction of the circulation of thiamine in the intracellular space $[4,6,9,11]$.

Thiamine responsive deficiency has been reported in other inborn errors of metabolism that lead to neurological diseases [11]. Genetic disorders of thiamine metabolism that lead to neurological diseases can be treated with highdose thiamine $[11,12,13]$. Recently, good results in sporadic degenerative diseases have been achieved with the same treatment [9]. The exact mechanism of thiamine responsiveness in these patients is unknown.

This clinical observation led to suppose that symptoms featuring dystonia could derive from a focal thiamine deficiency that determines a selective neuronal dysfunction. In other words, TD could have an important role in the pathogenesis of the disease symptoms. High doses of intramuscular thiamine increase the intracellular passive transport of the thiamine. Symptoms decrease when the glucose metabolism and other thiamine-dependent processes are led back to physiologic levels.

In this work, the patients reported a general improvement of the voluntary mobility and nonmotor symptoms. The patients continued the therapy after the three months reported in this work and both of them maintaining the same beneficial effects and, importantly, without any side effects. Indeed, there is any evidence in literature of thiamine-related adverse effects, even at high doses and for very long periods of 
time. $[8,14,15,16]$

We believe that the therapy may play an important role in limiting the progression of the disease and we deem necessary a lifelong use of high-dose thiamine in affected subjects. Lastly, also etiologically-diverse dystonia could be responsive to high doses of thiamine. [8,9,15]

In conclusion, we believe that this report represents an important contribution to the subject, nonetheless further experience is necessary to confirm the present observation.

\section{LEARNING POINTS}

The treatment with thiamine is immediately available for the treatment of DYT1

In literature, there is no study that observed side effects linked to daily use of high-dose thiamine.

We believe that this report opens a ray of hope for therapy of DYT1.

\section{ACKNOWLEDGEMENTS}

The authors thank Iara Tundo for the collaboration.

\section{REFERENCES}

[1] Albanese A, Asmus F, Bhatia K P, Elia AE, Elibol B, Filippini $\mathrm{G}$ et al. EFNS guidelines on diagnosis and treatment of primary dystonias. European Journal of Neurology 2011. 18: 5-18

[2] Kamm C. Genetics of dystonia. Fortschr Neurol Psychiatr. 2009 Aug;77 Suppl 1:532-6

[3] Lonsdale D. Thiamine and magnesium deficiencies keys to disease. Med Hypotheses. 2015 Feb; 84(2): 129-41.

[4] Parkhomenko Y.M., Pavlova, A.S. \& Mezhenskaya, O.A. Mechanisms Responsible for the high Sensitivity of Neural Cells to Vitamin B1 Deficiency. Neurophysiology. 2016. Dec. 48:6:429-448.

[5] Mkrtchyan S, Graf A, Bettendorff L, Bunik V. Cellular thiamine status is coupled to function of mitochondrial 2-oxoglutarate dehydrogenase. Neurochem Int 2016 Dec; 101:66-75.
[6] Costantini A, Pala MI, Colangeli M, Savelli S. Thiamine and spinocerebellar ataxia type 2 . BMJ Case Rep. Jan 10; 2013.

[7] Costantini A, Trevi E, Pala MI, Fancellu R. Thiamine and dystonia 16. BMJ Case Rep 2016 Jul 22; 2016

[8] Costantini A, Pala MI, Trevi E, Fancellu R.(2016) Thiamine and dystonia. J Transl Sci 3.

[9] Wallace DC, Murdock DG. Mitochondria and dystonia: The movement disorder connection? Proc Natl Acad Sci USA 1999 Mar 2; 96(5): 1817-1819

[10] Comelia CL, Leurgans S, Wuu J, Stebbins GT, Chmura T; Dystonia Study Group. Rating scales for dystonia: a multicenter assessment. Mov Disord. 2003 Mar; 18(3):303-12

[11] . Kono S, Miyajima H, Yoshida K, Togawa A, Shiragawa K, Suzuki H. Mutation in a Thiamine-Transporter Gene and Wernicke'slike Encephalopathy, $N$ ENGL J MED 360;17 NEJM.ORG APRIL 23, 2009.

[12] Serrano M, Rebollo M, Depienne C, Rastetter, Fernandez-Alvares E, Muchart $\mathrm{J}$ et al. Reversible generalized dystonia and encephalopathy from thiamine transporter 2 deficiency. Mov Disord. 2012 Sept 1; 27(10):1225-8.

[13] Ortigoza-Escobar JD, Serrano M, Molero M, Oyarzabal A, Rebollo M, Muchart J et al. Thiamine transporter-2 deficiency: outcome and treatment monitoring. Orphanet J Rare Dis. 2014 Jun 23;9:92.

[14] Van Dangen S, Brown RM, Brown GK, Thorburn DR, Boneh A. Thiamine-Responsive and non-responsive Patients with PDHC-E1 Deficiency: A Retrospective Assessment. JIMD Rep. 2015; 15:13-27.

[15] Thompson VB, Jinnah HA, Hess EJ. Convergent mechanisms in etiologicallydiverse dystonias. Expert Opin Ther Targets. 2011 Dec; 15(12):1387-403.

[16] Smithline HA, Donnino M, Greenblatt DJ, Pharmacokinetics of high-dose oral thiamine hydrochloride in healthy subjects. BMC clinical pharmacology 2012, 12:4.

Citation: Costantini Antonio, Pala Maria Immacolata, Trevi Erika, Maurini Serena, Vecchietti Stefania, Fancellu Roberto. Thiamine Therapy for Dystonia Type 1. ARC Journal of Neuroscience. 2018; 3(1):1-4. doi:dx.doi.org/10.20431/2456-057X.0301001.

Copyright: () 2018 Authors. This is an open-access article distributed under the terms of the Creative Commons Attribution License, which permits unrestricted use, distribution, and reproduction in any medium, provided the original author and source are credited. 